\title{
Reduction of CCD observations made with a scanning Fabry-Perot interferometer. III. Wavelength scale refinement
}

\author{
A. V. Moiseev \\ Special Astrophysical Observatory, Russian Academy of Sciences, Nizhnij Arkhyz, 369167, Russia
}

August 10, 2015/Revised: Neptember 7, 2015

\begin{abstract}
We describe the recent modifications to the data reduction technique for observations acquired with the scanning Fabry-Perot interferometer (FPI) mounted on the 6-m telescope of the Special Astrophysical Observatory that allow the wavelength scale to be correctly computed in the case of large mutual offsets of studied objects in interferograms. Also the parameters of the scanning FPIs used in the SCORPIO-2 multimode focal reducer are considered.
\end{abstract}

\section{Introduction}

Integral-field (3D) spectroscopy is now used extensively both in optical and IR observations on telescopes of various sizes. The most popular instruments employed are integral-field spectrographs and units (IFU) equipped with microlens arrays, fiber bundles, or slicers. However, the field of view provided by this technique barely reaches about one arcminute even in the best cases MUSE (Bacon et al., 2014) on the 8-m VLT telescope or PPAK/PMAS (Kelz et al., 2006) on the 3.6-m Calar Alto telescope. This limitation is due to the need to simultaneously record many spectra with sufficient spatial sampling. At the same time, the spectrometers based on scanning Fabry-Perot interferometer (FPI) mounted on 2-10$\mathrm{m}$ telescopes prove to be unchallenged when it comes to a combination of such parameters as a large (several arcminutes) field of view with spatial sampling similar to that of direct images and a relatively high $(R=\lambda / \delta \lambda>10000)$ spectral resolution (Boulesteix, 2002). The downside of these advantages is the narrow spectral interval (usually less than $50 \AA$ ) and the need to successively record individual interferogram frames (scanning or multiplex spectrometer). Account should also be taken of atmospheric transparency and seeing variations over long scanning cycles, which can often continue for several hours. Two approaches are employed to address these problems: the use of photon counting detectors, which allow multiple repeated scannings with short exposures, or the "slow" scanning with a high quantum efficiency CCD with finite readout time and noise levels. An example of the first approach is the $\mathrm{GH} \alpha \mathrm{FaS}$ instrument mounted on the $4.2-\mathrm{m}$ WHT telescope (Hernandez et al., 2008) and equipped with a GaAs photo-cathode and a microchannel plate based am-

Send offprint requests to: A. Moiseev e-mail: moisav@sao.ru plifier at the input of a commercial CCD. The second approach, the one involving a classic CCD, allows the same detector to be used for other types of observations such as direct imaging and long-slit spectroscopy, i.e., incorporating the FPI into a multimode instrument-the focal reducer. It is important to develop an observing and data reduction technique involving proper correction of atmospheric modulations in the case of slow scanning with an FPI. This approached is implemented on the 6-m telescope of the Special Astrophysical Observatory of the Russian Academy of Sciences (SAO RAS), where the FPI has been used as one of the operation modes of the SCORPIO focal reducer since 2000 (Afanasiev \& Moiseev, 2005).

For a detailed description of the data reduction technique used in observations with the FPI mounted on SCORPIO, see our previous papers of this series (Moiseev, 2002; Moiseev \& Egorov, 2008). In this paper we describe further progress in the application of this technique on the 6-m telescope for studies of ionized gas kinematics in Galactic and extragalactic objects. For instance, in 2010 first light was achieved on the SCORPIO-2 second-generation multimode focal reducer Afanasiev \& Moiseev, 2011), which includes the FPI mode. Since 2013 all such observations on the $6-\mathrm{m}$ telescope have been performed with SCORPIO-2 exclusively. Two new scanning piezoelectric interferometers were acquired, and the spectral resolution has been almost doubled.

In the first part of this paper (Section 2), we describe the parameters of the FPIs used on SCORPIO-2. The emergence of new hardware and the diversification of research tasks required a modification of the data reduction algorithm. Section 3 discusses the factors that cause wavelength scale errors in the case of frame-to-frame offsets of studied objects in interferograms. Section 4 describes 


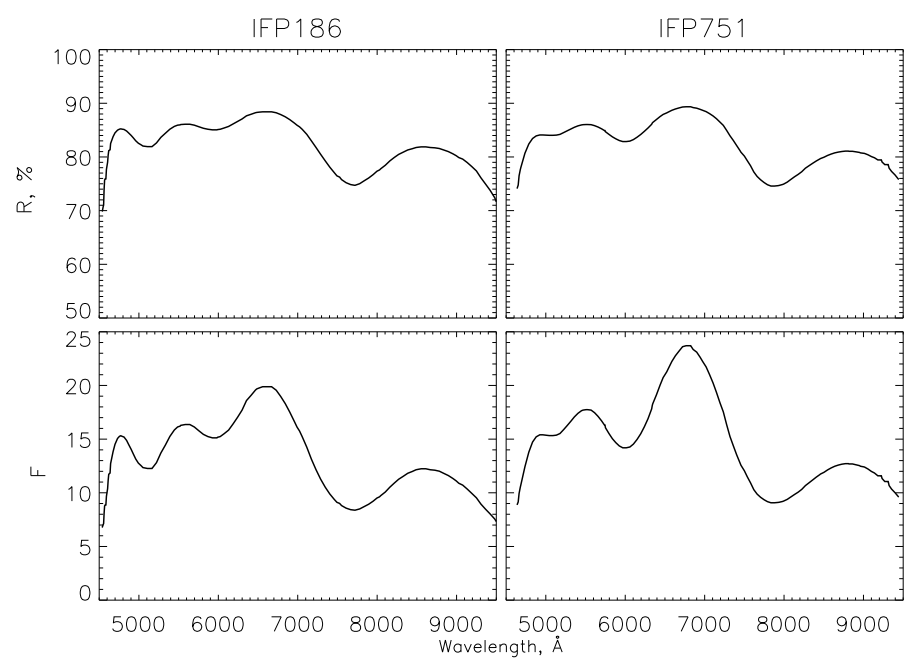

Fig. 1. Wavelength dependence of the reflection coefficient $R$ of interferometer plates (top) and the corresponding computed finesse $F$ (bottom). The left and right panels show the data for the IFP186 and IFP751 interferometers respectively.

a method for correcting this effect: such a correction is important for precise ionized gas velocity dispersion measurements. The concluding section discusses possible applications of the method developed.

\section{New Fabry-Perot interferometers at SAO RAS}

The principal parameters of FPI observations are practically identical both for SCORPIO and SCORPIO-2: the same field of view $\left(6 !^{\prime} 1 \times 6.1\right)$ with a $0.36 /$ pixel scale (in the case of $2 \times 2$ binned CCD readout). The differences include a somewhat higher sensitivity of the E2V-4240 CCD in the $\mathrm{H} \alpha$ region (95\% compared to $78 \%$ for the EEV $42-40$ CCD used in the old spectrograph). SAO RAS also acquired two new ET-50 scanning piezoelectric interferometers with serial numbers FS-1064 and FS-1081 (manufactured in 2009 and 2012 respectively) from IC Optical Systems, Ltd 1 . In the SCORPIO-2 nomenclature these interferometers are referred to as IFP186 and IFP751 respectively. The table1 lists the main parameters of the devices: $n$, the order of interference at the given wavelengths; $\Delta \lambda$, the free spectral range between two adjacent orders; $\delta \lambda$, the spectral resolution (FWHM of the line profile of the calibration lamp); $F$, the finesse; $n_{z}$, the number of images (channels) in the spectral interval. The last parameter is set during observations so as to ensure the minimum allowable sampling, i.e., $n_{z} \geq 2 F$.

These parameters were measured directly with SCORPIO-2 from the spectrum of the He-Ne-Ar calibration lamp with individual lines separated using narrowband filters. The $\delta \lambda$ parameter in the vicinity of $\mathrm{H} \alpha$ was estimated from the NeI $\lambda 6598.95$ line, and that in the vicinity of the $[\mathrm{O} \mathrm{III}]$ line-by simultaneously fitting the

\footnotetext{
1 http://www.icopticalsystems.com/
}

profiles of five Ne I + Ar II lines in the 5145-5152 A wavelength interval. The manufacturer provided the plot of the measurements of the reflection coefficient $R$ of interferometer plates as a function of wavelength (Fig. 1] top panels). Also shown in the same figure are the corresponding finesse characterizing the spectral resolution at different wavelengths as determined by formula

$F(\lambda)=k F_{0}(\lambda)$

Here $F_{0}(\lambda)$ is the finesse of "ideal" interferometer (with no defects and absolutely plane plates) computed by the following formula, known from FPI theory:

$F_{0}(\lambda) \approx \frac{\pi \sqrt{R(\lambda)}}{1-R(\lambda)}$

and the normalization factor $k$ chosen so as to ensure the best agreement between the resulting plot and both our measurements (see Table) and manufacturer's data $(F=$ 18 at $633 \mathrm{~nm}$ for both interferometers): $k=0.78$ and $k=$ 0.85 for IFP186 and IFP751 respectively. Figure 1 shows that observations with a sufficiently high finesse $(F>10)$ can be performed not only near the $\mathrm{H} \beta-[\mathrm{O}$ III $] \lambda 5007$ and $\mathrm{H} \alpha-[\mathrm{S} \mathrm{II}] \lambda \lambda 6717,6731$ lines but also in the vicinity of the Ca II $\lambda \lambda 8498,8542,8662$ triplet. This possibility was incorporated intentionally so that the instrument could be used to study stellar population kinematics by analyzing absorption lines.

We provisionally computed the order of interference $n$ for a given wavelength based on the gap between the interferometer plates as measured by the manufacturer (with an accuracy of about $3 \mu \mathrm{m}$ ). We then refined this quantity by measuring the positions of neighbouring (with a wavelength difference of $2-3 \Delta \lambda$ ) lines in the spectrum of the calibration lamp so that the wavelength difference measured after scanning would be as close as possible to value from the literature. For measurements with the available narrow-band filters and the He-Ne-Ar lamp, we selected line pairs in the 5080-5220 $\AA$ interval. The accuracy of such gap measurements is equal to $n \pm 1$ (about $\lambda / 2$ ), which is quite sufficient for further wavelength scale calibration in observations involving a sole calibrating line. Notice that during the first observations performed with IFP186, the order of interference was estimated as $n(\lambda 6563)=186$. It was later refined to be 188 , but the nomenclature was left unchanged.

Table 1. Parameters of the scanning FPIs used with SCORPIO-2

\begin{tabular}{l|c|c|c|c}
\hline Parameter & \multicolumn{2}{|c|}{ IFP186 } & IFP751 & IFP501 \\
& $\lambda 6563$ & $\lambda 5007$ & $\lambda 6563$ & $\lambda 6563$ \\
\hline$n$ & 188 & 246 & 751 & 501 \\
$\Delta \lambda, \AA$ & 34.9 & 20.3 & 8.7 & 13.1 \\
$\Delta \lambda, \mathrm{km} \mathrm{s}^{-1}$ & 1696 & 1216 & 399 & 598 \\
$\delta \lambda, \AA$ & 1.7 & 2.0 & 0.44 & 0.80 \\
$\delta \lambda, \mathrm{km} \mathrm{s}^{-1}$ & 78 & 120 & 20 & 36 \\
$F$ & 21 & 10 & 20 & 16 \\
$n_{z}$ & 40 & 30 & 40 & 36 \\
\hline
\end{tabular}




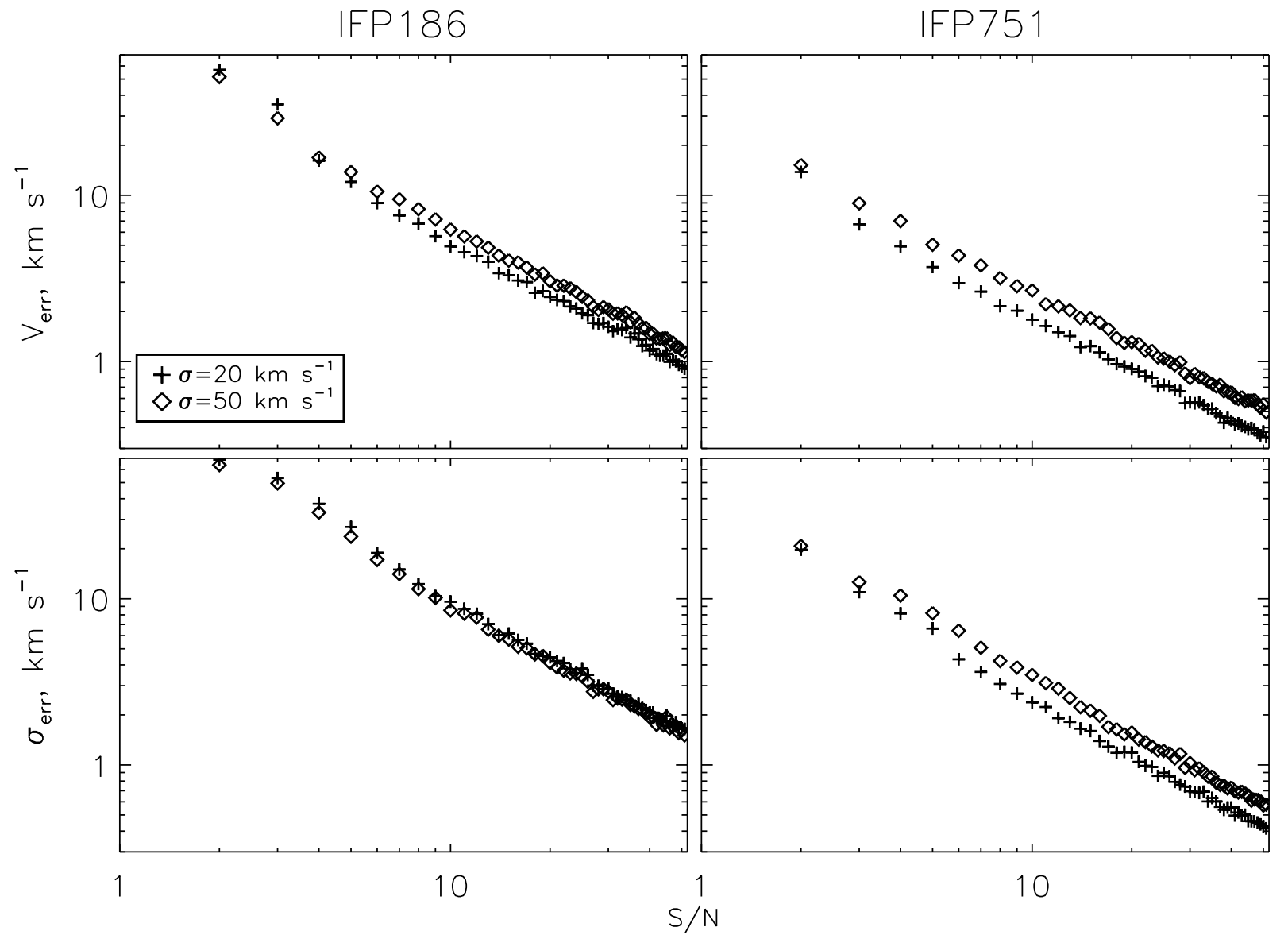

Fig. 2. Simulation of the measurement errors of kinematic parameters (at the $1 \sigma$ level) in the case of the Voigt-profile approximation of the $\mathrm{H} \alpha$ line: the dependence of the error of the measured radial-velocity (top) and velocity dispersion (bottom) on the signal-to-noise ratio. The corresponding data are provided both for the IFP186 (left) and IFP751 (right) interferometers. The crosses and diamond signs show the results of calculations for velocity dispersions of 20 and $50 \mathrm{~km} \mathrm{~s}^{-1}$ respectively.

For comparison, we also list in the table 1 the parameters of the old interferometer IFP501. It is practically hardly ever used now, because IFP751 provides almost twice better spectral resolution for a similar $\Delta \lambda$. IFP186 is more convenient for observations of faint objects with a wide range of radial velocities. In particular, it is used for observations of active galactic nuclei in the [O III] $\lambda 5007$ line.

Figure 2 shows the estimated measurement accuracy for main emission-line kinematic parameters, the radial velocity $v$ and velocity dispersion $\sigma$, plotted as functions of the signal-to-noise ratio $S / N$. Using a technique described in Moiseev \& Egorov (2008) we modeled the instrumental profile of the FPI by the Lorentz function with the FWHM adopted from the table 1 for the case of $\mathrm{H} \alpha$ observations. The wavelength scale parameters $\left(\Delta \lambda\right.$ and $\left.n_{z}\right)$ were also adopted from the table 1. We smoothed the profile by a Gaussian with a given dispersion $\sigma$, then superimposed noise and estimated $v$ and $\sigma$ by approximating the line by the Voigt profile. We performed 1000 independent trials for each fixed $S / N$. The figure shows the measurements made for velocity dispersions $\sigma=20$ and $50 \mathrm{~km} \mathrm{~s}^{-1}$, which corresponds to the typical range of this parameter in $\mathrm{H} \mathrm{II}$ regions in galaxy disks (see, e.g. Moiseev et al., 2015). It is evident from the figure that for the FPIs employed, the measurement accuracy for both kinematic parameters is better than $2-4 \mathrm{~km} \mathrm{~s}^{-1}$ for $S / N>20$.

SCORPIO-2 observations with the FPI are performed with FWHM = 13-35 ^-wide narrow-band filters cutting a narrow spectral interval around the line considered. Observations in the [O III] $\lambda 5007$ and $\mathrm{H} \alpha$ lines can be performed for objects with radial velocities ranging from -300 to $13000 \mathrm{~km} \mathrm{~s}^{-1}$. The filter set is systematically upgraded with the actual list available on the web page of the instrument 2

\section{Wavelength scale errors}

The estimates shown in Fig. 2 are the lower limits because they refer to the ideal case of uniform noise, which fails in the presence of bright telluric emission lines. Subtraction of night-sky lines may produce artifacts in reduced spectra. It is also important how accurately the instrumental

\footnotetext{
${ }^{2}$ http://www.sao.ru/hq/lsfvo/devices/scorpio-2/
} 
profile of the interferometer is reproduced during scanning. For a discussion of how the choice of a subtraction algorithm affects the resulting spectrum see Moiseev (2002). Below we analyze the factors that cause errors in the wavelength scale of observed spectra, which also depend on the adopted reduction technique.

Hereafter by "channels" we mean the individual twodimensional interferograms that make up the observed data cube $I(x, y, z)$, where $x, y$ are the CCD coordinates, and $z$ is the channel number proportional to the gap between the FPI plates. The reduction sequence can be subdivided into the following main steps (see Gordon et al., 2000; Moiseev, 2002, for details).

(1) Assembling the data cube from observed interferograms $I(x, y, z)$, which includes standard CCD frame reduction procedures: bias and dark frame subtraction and flat-fielding

(2) Channel-by-channel airglow line subtraction.

(3) Channel correction based on the photometry of field stars: compensation of mutual frame offsets, atmospheric transparency and seeing variations.

(4) Conversion of the spectra to the wavelength scale, i.e., transition to the $I(x, y, \lambda)$ data cube. If a photon counter is used, the sky background subtraction can be performed after this stage (Daigle et al., 2006).

Conversion to the wavelength scale is conveniently performed based on the "phase offset" map $p(x, y)$, which shows the positions of the line center in the cube made up of the interferograms of the selected line in the spectrum of the calibrating lamp $\left(\lambda_{\text {calib }}\right)$. Figure 3 shows examples of such maps for the high- and low-resolution SCORPIO-2 interferometers. Phase offset $p$ varies almost quadratically with the distance $r$ from the center of the rings. Sharp discontinuities correspond to the change of the interference order. If the phase map is based on the measurements of a calibration lamp line that is close (in terms of $\Delta \lambda$ ) to the spectral line of the observed object, then in practice the transition $I(x, y, z) \rightarrow I(x, y, \lambda)$ reduces, up to a certain constant, to shifting each spectrum in the cube by $-p(x, y)$.

Problems with accurate wavelength scale calibration arise in the case of substantial mutual image offsets between individual channels. These offsets are primarily due to instrumental flexures, because the position of the telescope and the parallactic angle may change appreciably over several hours of observations while all channels are scanned.

Each channel contains a mixture of spatial and spectral information, because $\lambda$ decreases with $r$ (Fig. 4). Angular dispersion increases linearly with radius and is independent of the interference order. It can be easily shown that

$\frac{d \lambda}{d \vartheta}=-\lambda \tan \vartheta$ where $\vartheta$ is the distance from the center in angular units. The dispersion expressed in units of scanning steps is proportional to the order of interference:

$\frac{d z}{d \vartheta}=\frac{n_{z}}{\Delta \lambda} \frac{d \lambda}{d \vartheta}=-n n_{z} \tan \vartheta$

as illustrated by the bottom plot in Fig. 3. In observations with IFP751, mutual channel offsets of about one pixel $(0.7)$ prove to be critical even for objects in the central part of the frame $\left(r \leq 100^{\prime \prime}\right)$. In this case the formal application of channel-by-channel correction procedures (stage 3 ) shift the data point in the spectrum by one channel.

Such offsets usually do not exceed 0.5 pixel over twoto-three scanning hours. However, they exceeded 1-2 pixels in some cases (play in the carriage of the SCORPIO dispenser). The adopted observing sequence with the odd channels recorded first and then followed by even channels partially removes the effect due to monotonic variation of offsets with time. However, these offsets may nevertheless affect at least the width of the resulting line profile.

\section{4. $\Lambda$-cube based correction}

We proposed the following modification of the data reduction algorithm to avoid the above problems. Instead of the two-dimensional phase map our new procedure uses the $\Lambda(x, y, z)$ cube (hereafter referred to as the $\Lambda$-cube) that stores the wavelength values for each data point in $I(x, y, z)$ :

$\Lambda(x, y, z)=[z-p(x, y)] \frac{\Delta \lambda}{n_{z}}+\lambda_{\text {calib }}$

Figure 5 shows an example of such a cube. In practice, wavelengths can be conveniently expressed in fractions of the scanning step (the bracketed expression in formula (5) ). The $z=n_{z}$ quantity is added to each successive order, and hence the resulting $\Lambda$-cube contains no discontinuities due to the change of the interference order.

If $\delta x, \delta y$ channel offsets have to be applied to the object cube $I(x, y, z)$ in the process of primary reduction, then the same offsets should also be applied to the corresponding channels of the $\Lambda$-cube (correction based on field stars, compensation of the offset between the calibration cube and test interferograms of the rings from the calibration lamp before and after observations of the object (Moiseev, 2002)). Each channel $I(x, y, z)$ thus stores correct wavelengths corresponding to each image pixel, up to interpolation errors. The $I(x, y, z) \rightarrow I(x, y, \lambda)$ conversion is performed by interpolating the observed intensities at each point $(x, y)$ from the $\Lambda$-cube scale to a uniform wavelength grid.

Figure 6] compares ionized gas velocity dispersion measurements in three nearby dwarf galaxies from Moiseev et al. (2015) performed using the phase map $(\sigma($ old $))$ and the $\Lambda$-cube method $(\sigma($ new $))$. Shown are the cases where mutual channel offsets exceeded one pixel for technical reasons. Usually $\sigma($ new $)<\sigma($ old $)$, because in 

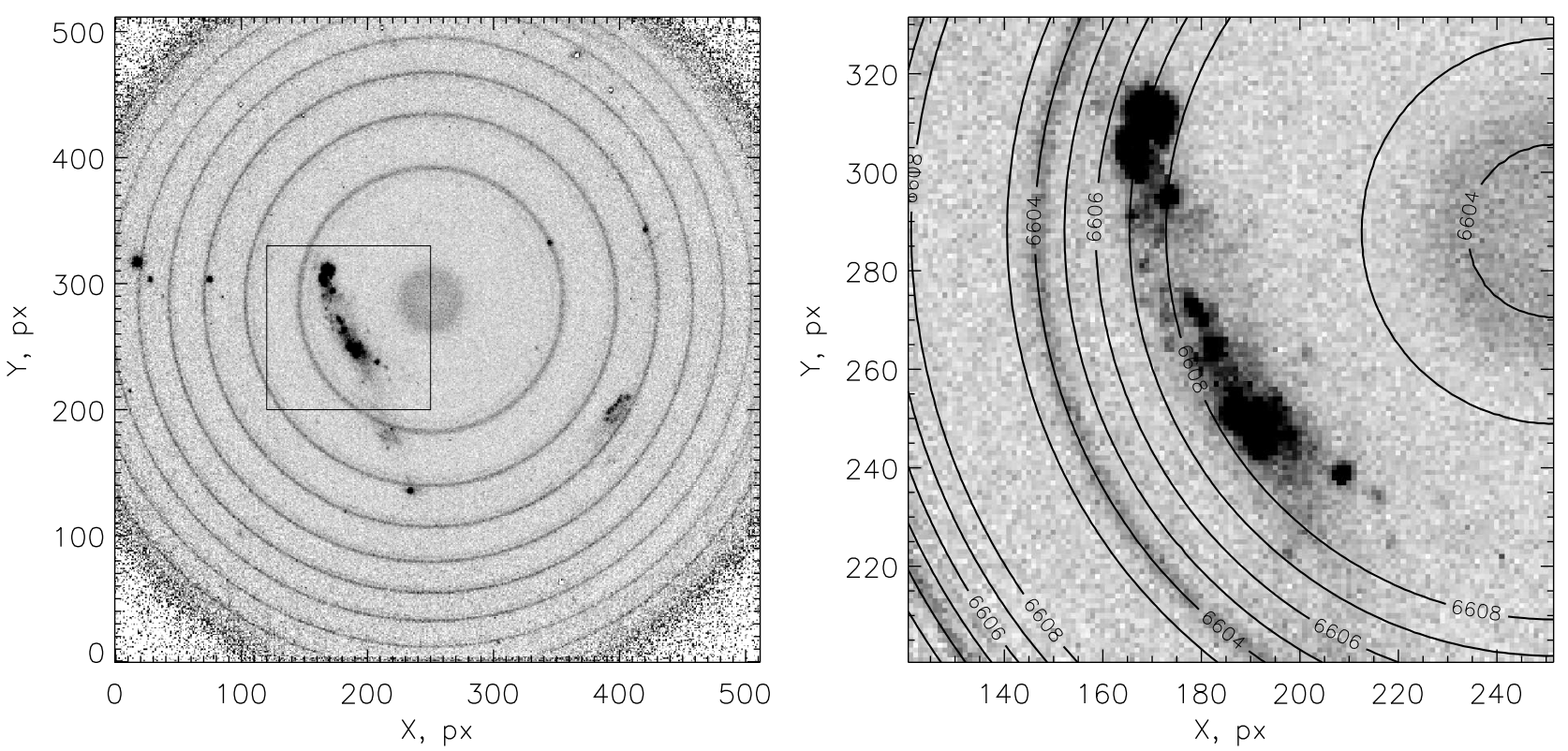

Fig. 4. H $\alpha$ observations of the galaxy UGC 260 with IFP751 mounted on SCORPIO-2. Left: an example of a frame with both the emission from the galaxy and the rings from the $\lambda 6604$ airglow line are immediately apparent. The square area is shown in the right panel, the contours indicate the wavelength scale with a contour step of $1 \AA$.

the case of a nonzero offset the phase map method introduces extra scatter in $\lambda$ resulting in a broadening of measured lines. However, line profile distortions may be even more intricate, because they depend on the position of the target relative to the optical axis, direction and magnitude of the offset, etc. That is why $\sigma$ (new) $>\sigma$ (old) in some areas. However, in the cases of channel offsets no greater than $1^{\prime \prime}$, the systematic error of velocity dispersion measurements usually does not exceed $10 \mathrm{~km} \mathrm{~s}^{-1}$, i.e., the width of the instrumental profile $\mathrm{FWHM} \approx 2.35 \sigma$. Notice that measured line barycenter positions are almost always free of systematic errors.

\section{Discussion}

Since 2011 the $\Lambda$-cube based calibration method has been incorporated into the IFPWID software package (Moiseev \& Egorov, 2008), written in IDL. It avoids emission-line profile distortions arising in the cases of individual channel offsets caused by technical problems with the guiding system or spectrograph mechanisms. Although, as shown above, the distortions lead mainly to the line profile broadening, they prove to be critical in the tasks where the aim is not just to determine the velocity field but also to perform a detailed study of the line profile. A number of studies carried out on the SAO RAS 6-m telescope in recent years belong to this category, e.g., the measurement of ionized gas velocity dispersion in dwarf galaxies (Moiseev et al., 2015) or the study of young stellar object outflows (Movsessian et al., 2015).
The proposed wavelength scale calibration method is not fundamentally new. Bland \& Tully (1989) pointed out in their classic paper the need for individual phase correction of the channels in the cases of ring center offsets. Mitchell et al. (2015) use a similar idea in their analysis of observations made with the FPI on the 10-m Southern African Large Telescope (SALT). In this case the wavelength distribution in each channel is constructed based on the preliminary measurement of the calibrating line (or the measurements of the rings from night sky emission lines) and the determination of several constants characterizing the appearance of the fringe pattern (see also Gordon et al., 2000).

At the same time, the use of the $\Lambda$-cube makes our reduction programs easily adaptable to the analysis of the data obtained with FPIs on other telescopes. In particular, we used this technique to reduce the observations obtained with the scanning FPI mounted on SALT (Brosch et al., 2015). The fundamental difference from observations performed at SAO RAS is that because of various technical limitations on the exposure duration, the data cube cannot be scanned over the entire overlap-free $\Delta \lambda$ range but only in the region where the location of an emission line is expected.

We hope that the parameters of the interferometers used with SCORPIO-2 and the modifications of the data reduction technique described in this paper would be useful for colleagues interested in the study of the kinematics of extended objects with the scanning FPI on the SAO RAS 6-m telescope. 

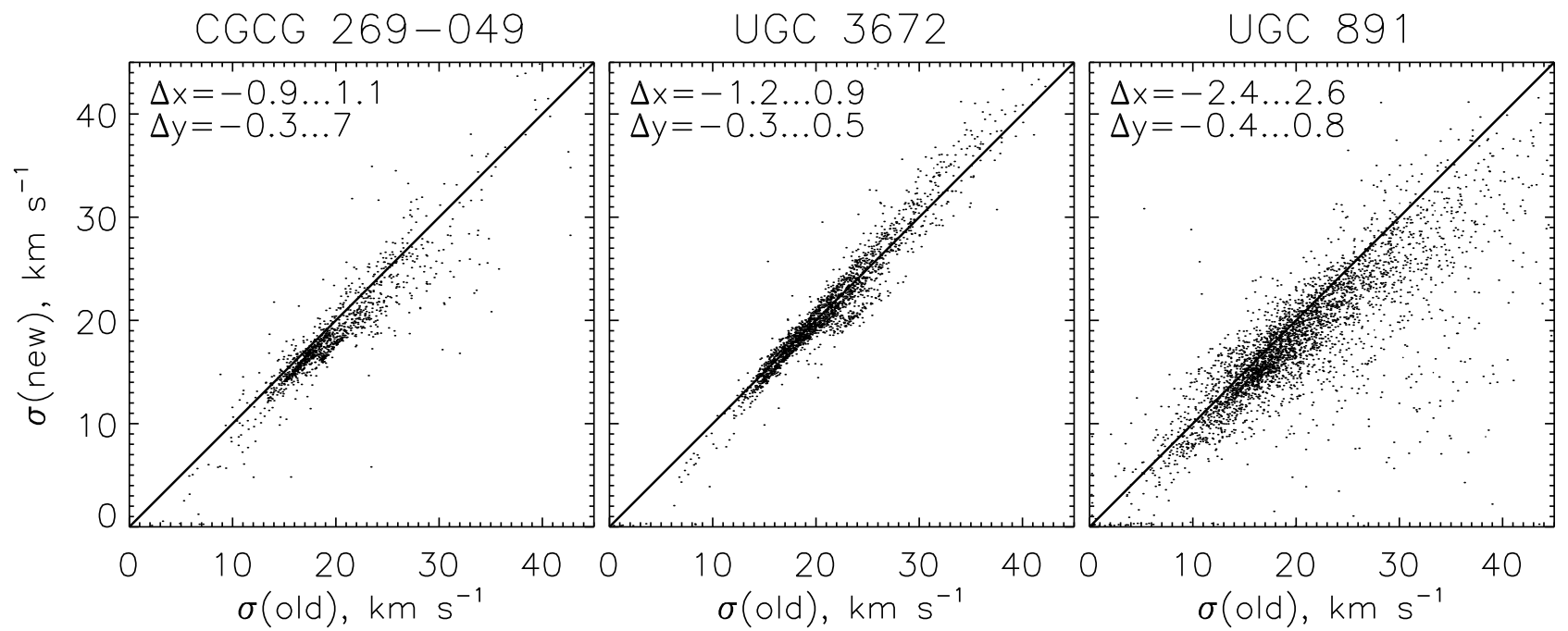

Fig. 6. Comparison of $\mathrm{H} \alpha$ velocity dispersion measurements for ionized gas in three dwarf galaxies (their names are given at the top), made with IFP751. The wavelength scales along the horizontal and vertical axes are based on the phase map $\sigma$ (old) and the $\Lambda$-cube $\sigma$ (new) respectively. The dots show the measurements in each data cube pixel. The solid lines corresponds to the bisector $\sigma(\mathrm{old})=\sigma($ new $)$. The numbers in the top left corner indicate the range of mutual channel offsets in pixels along the $x$ and $y$ axes relative to the mean position averaged over all observations. One pixel corresponds to $0 . ! 7$.

Acknowledgements. This work was supported by grant MD3623.2015.2 of the President of the Russian Federation and by the non-profit Dynasty Foundation. This paper is based on the observations carried out on the 6-m telescope of the Special Astrophysical Observatory, operated with the financial support of the Ministry of Education and Science of the Russian Federation (agreement No. 14.619.21.0004, project ID RFMEFI61914X0004). I am grateful to IC Optical Systems, Ltd. and personally to Chris Pietraszewski for sharing technical information and sustained interest in how their interferometers are used in observations on the 6-m telescope.
Mitchell, C. J., Williams, T. B., Spekkens, K., et al. 2015, AJ, 149, 116

Moiseev, A. V. 2002, Bulletin of the Special Astrophysics Observatory, 54, 74

Moiseev, A. V. \& Egorov, O. V. 2008, Astrophysical Bulletin, 63, 181

Moiseev, A. V., Tikhonov, A. V., \& Klypin, A. 2015, MNRAS, 449, 3568

Movsessian, T. A., Magakian, T. Y., Moiseev, A. V., \& Gevorgian, M. G. 2015, Astrophysical Bulletin, 70, 206

\section{References}

Afanasiev, V. L. \& Moiseev, A. V. 2005, Astronomy Letters, 31, 194

Afanasiev, V. L. \& Moiseev, A. V. 2011, Baltic Astronomy, 20, 363

Bacon, R., Vernet, J., Borisova, E., et al. 2014, The Messenger, 157, 13

Bland, J. \& Tully, R. B. 1989, AJ, 98, 723

Boulesteix, J. 2002, in Astronomical Society of the Pacific Conference Series, Vol. 282, Galaxies: the Third Dimension, ed. M. Rosada, L. Binette, \& L. Arias, 374

Brosch, N., Väisänen, P., Kniazev, A. Y., \& Moiseev, A. 2015, MNRAS, 451, 4114

Daigle, O., Carignan, C., Amram, P., et al. 2006, MNRAS, 367,469

Gordon, S., Koribalski, B., Houghton, S., \& Jones, K. 2000, MNRAS, 315, 248

Hernandez, O., Fathi, K., Carignan, C., et al. 2008, PASP, 120,665

Kelz, A., Verheijen, M. A. W., Roth, M. M., et al. 2006, PASP, 118, 129 

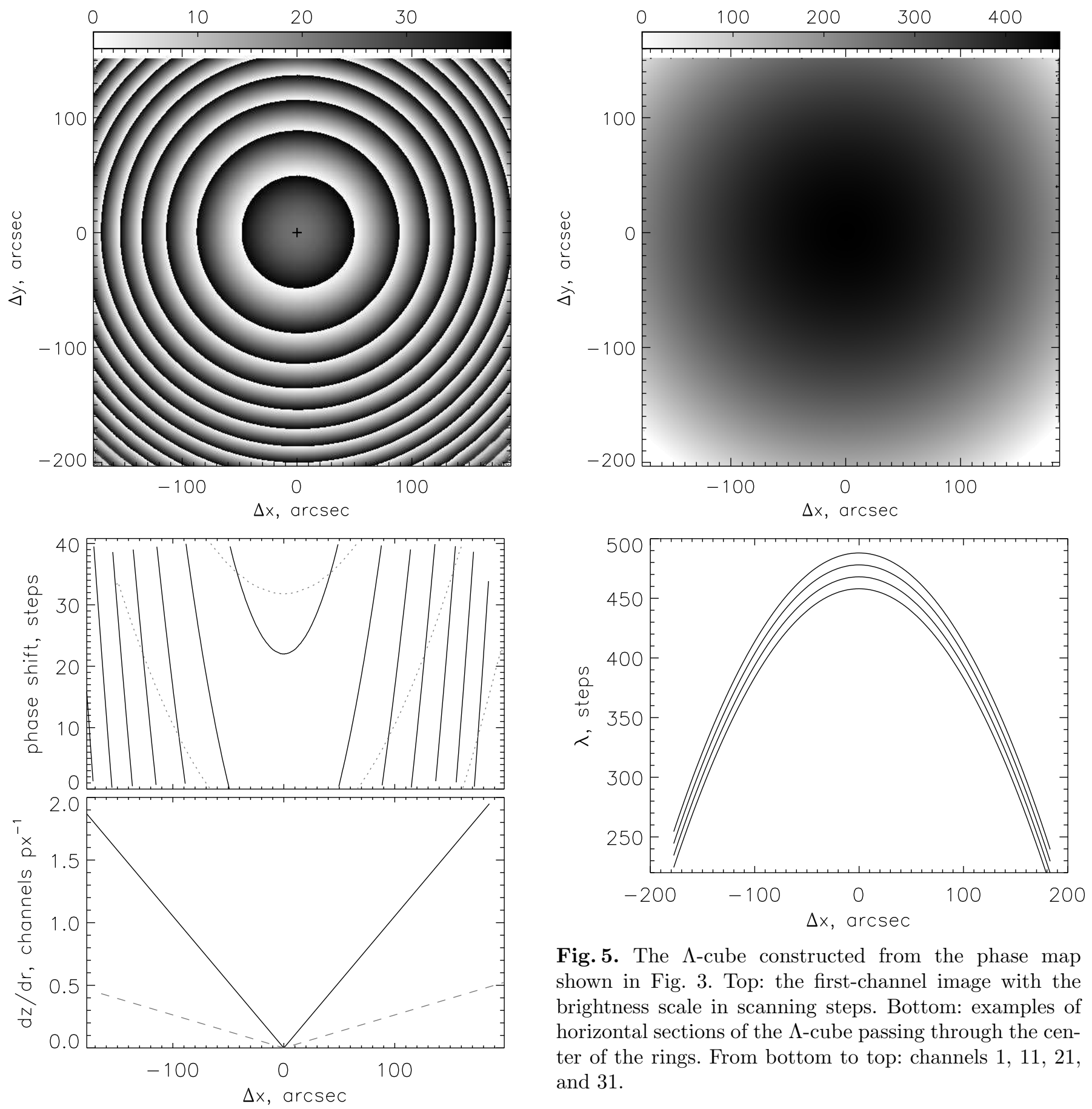

Fig. 5. The $\Lambda$-cube constructed from the phase map shown in Fig. 3. Top: the first-channel image with the brightness scale in scanning steps. Bottom: examples of horizontal sections of the $\Lambda$-cube passing through the center of the rings. From bottom to top: channels 1, 11, 21, and 31 .

Fig. 3. Top: a phase map based on the measurements of the Ne I $\lambda 6598.95$ line in the spectrum of the calibration lamp (in units of spectral channels for IFP751). Middle: horizontal sections of phase maps passing through the center of the fringe pattern for IFP751 (the solid line) and IFP186 (the dotted line). Bottom: the corresponding variations of angular dispersion along the radius (the same designations are used). 